Mitteilungen der Österreichischen Geographischen Gesellschaft,

154. Jg. (Jahresband), Wien 2012, S. 212-234

\title{
INFORMELLES WOHNEN ALS ROUTINe? Multiple urbane Transformationen in Der Agglomeration Belgrad: Das Beispiel KaluĐerica ${ }^{1)}$
}

Daniel GöLER, Bamberg, Marija Martinović, Ivan RAtKaJ und Danica ŠAntić, alle Belgrad [Beograd]*

mit 6 Abb. und 2 Tab. im Text

\section{INHALT}

Summary

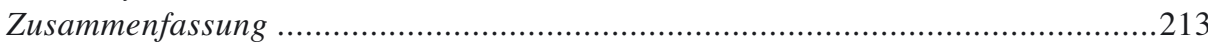

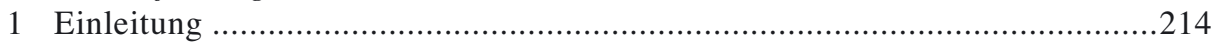

2 Informelle Siedlungen im post-jugoslawischen Transformationskontext.........215

3 Die sozialistische Urbanisierung und ihre Folgen für Jugoslawien

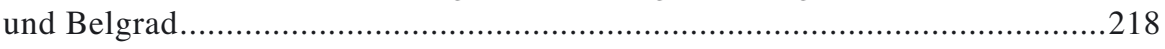

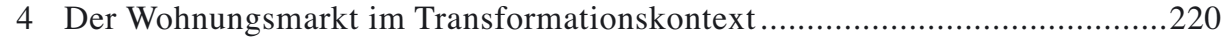

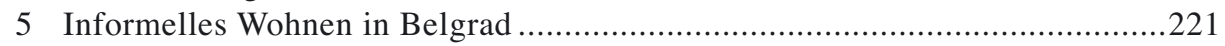

6 Das Exempel Kaluđerica - Informalität als Marginalität? ..............................222

7 Formalisierung und Legalisierung als Herausforderungen ............................2230

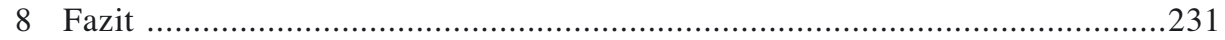

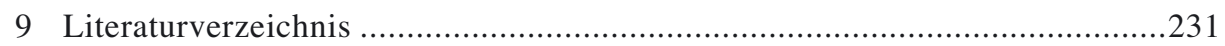

\footnotetext{
1) Die Studie wurde im Rahmen einer projektbezogenen Partnerschaft der Geographischen Institutionen der Universitäten Bamberg und Belgrad vom DAAD sowie vom Serbischen Wissenschaftsministerium (Projekt-ID 176017) dankenswerterweise finanziell unterstützt. Dank gilt zudem zwei anonymen Gutachtern für die wertvollen Hinweise zur Optimierung des Manuskriptes!

* Prof. Dr. Daniel GöLER, Institut für Geographie, Otto-Friedrich-Universität Bamberg, Am Kranen 1, D-96045 Bamberg; E-Mail: daniel.goeler@uni-bamberg.de, http://www.uni-bamberg.de/geographie; Marija Martinović, Dr. Ivan RatKAJ, PhD Assistent Danica Š ANTIĆ, alle Geographische Fakultät der Universität Belgrad, Studentski trg 1, 11000 Beograd; E-Mail: mmartinovic@gef.bg.ac.rs; ivan@gef.bg.ac.rs; danicam@gef.bg.ac.rs, http://www.gef.bg.ac.rs
} 


\section{Summary}

Informal housing as routine? Multiple urban transitions in the Belgrade metropolitan area (with Kaluđerica as an example)

Informal housing in Southeast Europe is not a new phenomenon by any means. The problem of informal settlements in Belgrade [Beograd] and the rest of post-Yugoslavia goes back to the 1960s, when growing Yugoslav cities suffered a massive housing shortage. Housing policies tolerated private, individual, and often informal construction activities of rural-urban migrants and low-medium income households. These activities continued and intensified during the late Socialist and early transitional periods. According to the 2002 masterplan of Belgrade, informal settlements covered more than $40 \%$ of the total residential area. As a result of these long-term, intensive informal developments, this paper concludes that its case study, Kaluđerica and the surrounding area, has grown to become one of the biggest informal settlements on the Balkans. This paper analyses these developments with the path-dependency approach. Sub-urbanisation of this kind is characterised by a high degree of informality and social marginality and differs greatly from both the well-known Western pattern, as well as the East-European pattern. Referring to the long-term development, informality is interpreted as a multiple (post-)Communist routine.

\section{Zusammenfassung}

Informelles Wohnen in Südosteuropa ist kein neues Phänomen. Im post-jugoslawischen Raum, so auch in Belgrad [Beograd], geht das Problem auf die 1960er Jahre zurück, als die wachsenden jugoslawischen Städte unter einem massiven Wohnungsmangel litten. Die nationale Wohnungspolitik duldete deshalb neben dem staatlichen Sektor private und oft informelle Bautätigkeiten von Zuwanderern aus ländlichen Regionen. Solche individuellen Aktivitäten setzten sich in der Transformationsperiode fort und intensivierten sich sogar. Im aktuellen Belgrader Stadtentwicklungsplan sind ca. $40 \%$ der Flächen mit Wohnfunktion als informelle Siedlungsbereiche ausgewiesen. Das hier exemplarisch betrachtete Kaluđerica im suburbanen Raum der Agglomeration Belgrad ist eine der größten Siedlungen dieser Art auf dem Balkan. Es ist das Ergebnis einer kontinuierlichen und intensiven informellen Siedlungsentwicklung, deren Aufarbeitung entsprechende Pfadabhängigkeiten berücksichtigen muss. Der vorgelegte Befund weicht von bekannten west- und osteuropäischen Suburbanisierungsmustern ab und ist ganz erheblich von städtebaulicher Informalität und sozialer Marginalität gekennzeichnet. Wegen seiner Langfristigkeit und der tiefen gesellschaftlichen Verwurzelung wird Informalität in diesem Sinn als multiple (post-)kommunistische Routine interpretiert. 


\section{Einleitung}

Probleme von Marginalsiedlungen und das Phänomen informellen Wohnens werden zumeist im Zusammenhang mit Entwicklungsländern betrachtet. Dort sind sie tatsächlich weit verbreitet; sie fallen ungemein facettenreich aus und sind in der geographischen Forschung entsprechend präsent. Das Thema lässt sich jedoch keineswegs auf diese Regionen begrenzen. Vielmehr handelt es sich um eine globale Erscheinung (vgl. UN-HABITAT 2003, S. 195ff.). Informelle Siedlungen finden sich in der Regel dort, wo staatliche Autorität und Zivilgesellschaft schwach ausgeprägt sind und, sei es aufgrund des Wachstums der Bevölkerungszahl oder des zunehmenden Wohlstandes, eine große Nachfrage nach Wohnraum herrscht. Die dabei wirkmächtigen Umbrüche in Staat, Politik, Wirtschaft und Gesellschaft sind, ceteris paribus, die Kernelemente des post-sozialistischen Transformationskontextes. Und so darf es nicht erstaunen, dass Informalität auch ein zentraler Bestandteil der Veränderungen in der ehemals sozialistischen Welt ist. Allerdings wird häufig übersehen, dass es beispielsweise mit spontanen und individuellen Bau- und Ausbautätigkeiten (ZEGARAC 1999 oder BouZAROVSKi, SALUKVADZE \& Gentile 2011), den weit verbreiteten Tauschwohnungsmärkten (SCHUlz 1991) oder den sowjetischen Kolchosmärkten (STADELBAUER 1991) vergleichbare individuelle Reaktionen auf Versorgungsmängel schon damals gegeben hat.

Unsere Argumentation zum Themenfeld informellen Wohnens unter den Bedingungen der post-sozialistischen Transformation, einem in der regionalen Transformationsforschung randlich behandelten Gegenstand, folgt in theoretisch-konzeptioneller Sicht der Berücksichtigung von Pfadabhängigkeiten (GrabHer \& STARK 1997; STARK 1992; SÝKORA 2008). Damit wird pointiert, dass ganze Sets von zementierten, nicht-kodifizierten Handlungsroutinen, deren Ursprünge in der sozialistischen (und manchmal in der vor-sozialistischen) Gesellschaft zu suchen sind, nach dem Systemwechsel vielfach fortgeführt und fallweise intensiviert wurden. Wir interpretieren solche Handlungsstränge - hier in Zusammenhang mit informellem Wohnen - als „Routinen“, welche aufgrund ihrer regionalen Unterschiedlichkeit kontextbezogen zu problematisieren sind (SÝ́KORA \& BouZARovski 2012, S. 5).

Am Exempel Kaluđerica, einer Stadtrandsiedlung in der Agglomeration Belgrad [Beograd] (Serbien), werden dazu Fragen der Herausbildung und der Dimension des Phänomens analysiert. Die zeitgeschichtlichen Wurzeln werden mit Elementen des post-sozialistischen Handlungskontextes kontrastiert. Auf dieser Basis werden die resultierenden städtebaulichen und stadtplanerischen Herausforderungen und Perspektiven aufgezeigt. Ziel der sowohl theoretisch als auch empirisch informierten Argumentation ist es, den Diskurs um Transformationen im urbanen Raum um die neue Perspektive der Interpretation städtebaulicher Informalität als (post-)sozialistische Routine zu bereichern. 


\section{Informelle Siedlungen im post-jugoslawischen Transformationskontext}

Informelles Wohnen ist in den Städten Südosteuropas keine Randerscheinung. Ein besonders spektakuläres Beispiel stellt sicherlich das albanische Tirana dar. Dessen Einwohnerzahl und Siedlungsfläche hat sich seit dem Systemwechsel in erstaunlich kurzer Zeit durch mehr oder weniger spontane Siedlungsnahmen von Zuwanderern aus allen Landesteilen vervielfacht (DOKA \& GöLER 2010). Die auf islamischem Gewohnheitsrecht basierenden Gecekondular zeigen dagegen deutlich mehr Kontinuitätslinien und entfalten als eine relativ kapitalarme Form des Siedelns auf okkupierten Flächen auch heute noch eine erhebliche Dynamik an den türkischen Stadträndern (HÜTTEROTH \& HöHFELd 2002, S. $310 \mathrm{ff}$.). Im (ehemaligen) Jugoslawien sind informelle Siedlungen sowohl ein systemübergreifendes großstädtisches Element als auch ein Transformationsphänomen, welches generell wieder an Bedeutung zu gewinnen scheint (dazu auch Göler \& Lehmeier 2011a, b; Bouzarovski 2011; Tsenkova 2010; Tosić 2010; Hirt 2009; Spevec \& Klempić Bogadi 2009).

Probleme des informellen Wohnungsbaus in Südosteuropa standen 2004 folgerichtig auf der Agenda einer Ministerkonferenz in Wien. Qua definitione wurde dort festgehalten:

"Informal settlements are human settlements, which for a variety of reasons do not meet requirements for legal recognition (and have been constructed without respecting formal procedures of legal ownership, transfer of ownership, as well as construction and urban planning regulations), exist in their respective countries and hamper economic development. While there is significant regional diversity in terms of their manifestation, these settlements are mainly characterized by informal or insecure land tenure, inadequate access to basic services, both social and physical infrastructure and housing finance." (Ministerial Conference on Informal Settlements in South EASTERN EUROPE 2004, S. 1).

Als charakteristische Elemente werden also erstens Fragen des Grundeigentums und zweitens Aspekte des Planungs- und Baurechts betont. Selbst wenn es keine international einheitlich gehandhabte Definition gibt, so führt das doch zu einer aus Sicht der Geographie akzeptablen Differenzierung: Danach ist das spontane Siedeln auf unrechtmäßig okkupiertem Land definitiv illegal; unter informell werden Fälle verstanden, in denen die Akteure zwar über einen Rechtstitel über das Grundstück verfügen, die Gebäude jedoch ohne behördliche Genehmigung errichtet worden sind (Herrle 2010, S. 232f.). In der Praxis sind die Grenzen oft fließend. Fehlende Grundstückskataster, im Detail nicht mehr nachvollziehbare Eigentumsübertragungen oder unklare, nicht existente oder nur lax umgesetzte Planungsvorgaben erschweren die exakte Abgrenzung.

Allein in Belgrad gibt es mehr als 50 solcher Siedlungen, in denen 9,3\% der Bevölkerung leben (vgl. Abb. 1). Neben den mäßig verdichteten informellen Stadtrandsiedlungen (wie Kaluđerica, vgl. Abb. 2) existieren sehr kompakte und teilweise sehr dicht bebaute Areale auf öffentlichen Flächen. Jener Typus sind - es sei der Vollständigkeit 


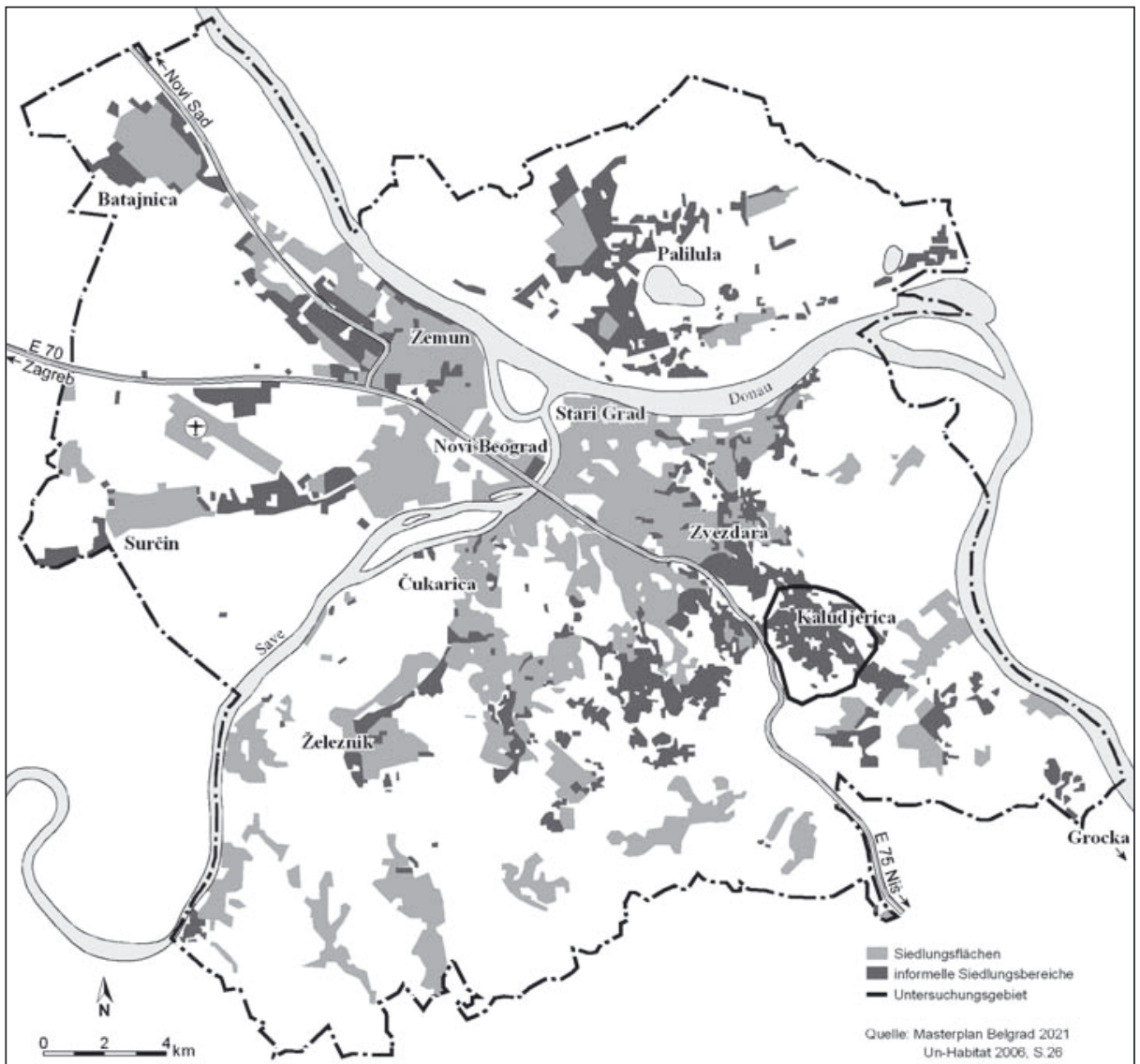

Abb. 1: Informelle Siedlungsbereiche in der Agglomeration Belgrad

halber erwähnt - meist Slum-ähnliche Siedlungen von Roma mit improvisierten, oft nur temporär bestehenden Wohneinheiten (UN-HABITAT 2006, S. 11). Sie finden sich in Belgrad zumeist in integrierten Lagen, beispielsweise in Parks, auf innerstädtischen Brachen oder Marginalflächen entlang von Bahnstrecken und Autobahnen. Zur Variationsbreite städtebaulicher Informalität in Belgrad gehören des Weiteren zahllose illegale Aneignungen und Umnutzungen von Gemeinschaftsflächen von Wohnanlagen in private Wohn- und Geschäftsräume oder ungeplante Anbauten an legale Wohn- und Geschäftsobjekte. Sie wurden additiv in die städtischen Strukturen eingefügt und okkupieren öffentlichen Grund.

Im hier behandelten Zusammenhang und im Hinblick auf das Wohnen manifestiert sich städtebauliche Informalität in fünf grundsätzlichen Problemfeldern, die eine gewaltige Herausforderung und eine wichtige Zukunftsaufgabe für die Belgrader Stadtplanung und -politik darstellen. Das beinhaltet insbesondere rechtliche, wirtschaftliche, städtebauliche, zivilgesellschaftliche und letztlich auch politische Komponenten (ILIĆ 


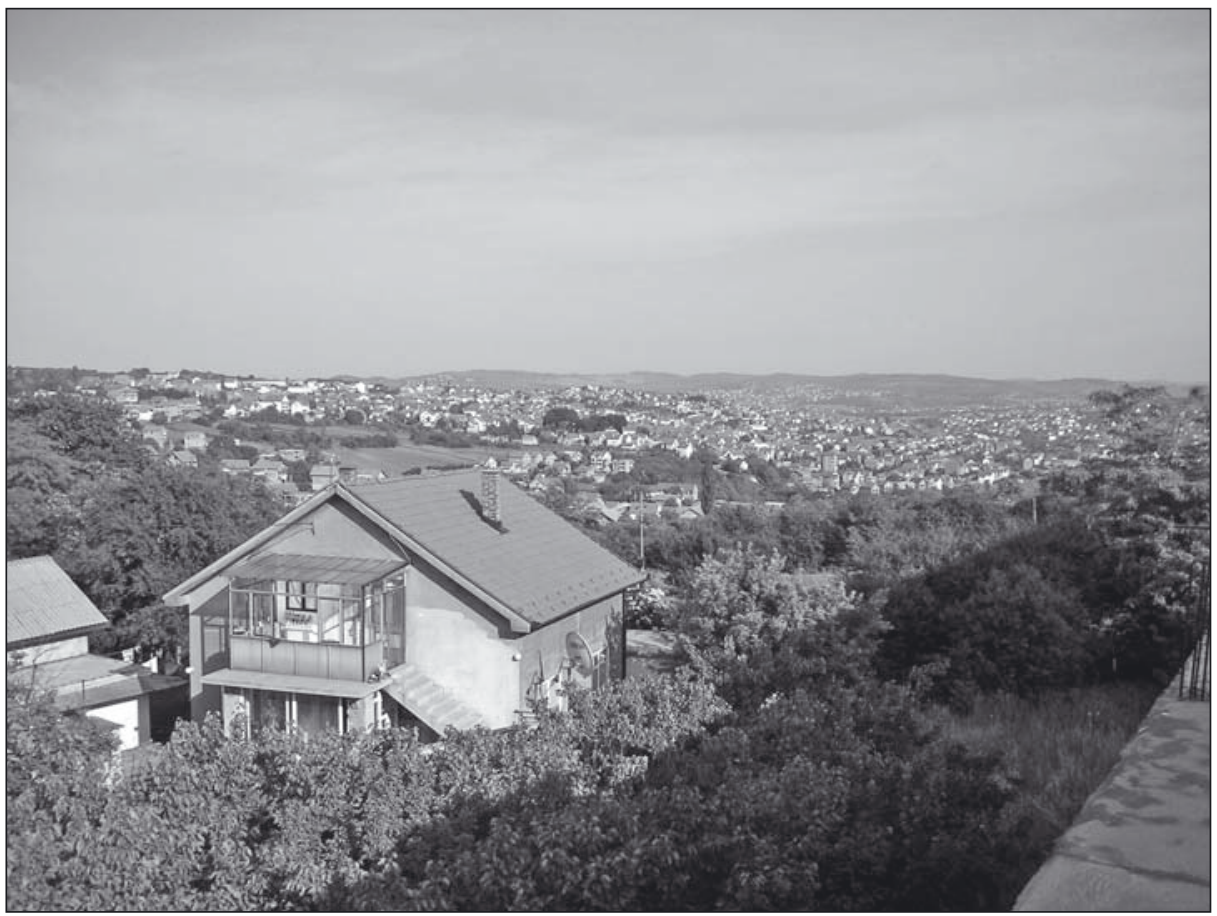

Abb. 2: Informelle Stadtrandsiedlung in der Agglomeration Belgrad - das Beispiel Kaluđerica (Aufnahme D. Göler 2005)

et al.2004). Rechtliche Aspekte (1) sind vor allem die unklaren Eigentumsverhältnisse. Da ein Rückbau im Regelfall nicht zur Debatte stehen kann, stellt sich die Frage einer nachträglichen Legalisierung. Erst die juristisch gesicherte Eigentümerschaft definiert Rechte und Pflichten, wie beispielsweise die Möglichkeit zur Erhebung von Steuern und Abgaben. Zum juristischen tritt also (2) ein ökonomisches Problem, denn informelle Siedlungen belasten einseitig die öffentliche Hand, ohne dass entsprechende Beiträge entrichtet werden. Über die Legalisierung ließe sich also das städtische Budget (und damit die Investitionsfähigkeit in die Infrastrukturversorgung) erhöhen und zugleich ein Immobilienmarkt schaffen. Das muss (3) in städtebaulicher Sicht mit der konsequenten Regulierung und strengen Kontrolle von Bauvorhaben anhand verbindlicher Planungsgrundlagen einhergehen.

Die Bewohner solcher Siedlungen leben in ständiger Unsicherheit und, oft unbewusst, in einem Stadium hoher sozialer Verwundbarkeit (4). Die Verbesserung der sozialen Sicherheit und Stabilität, die Minimierung von Nutzungs- und Nachbarschaftskonflikten und die Erhöhung der sozialen und infrastrukturellen Standards in den informellen Siedlungen ist eine der obersten Aufgaben der Zivilgesellschaft. Last but not least fehlen den Betroffenen jegliche politische Partizipationsmöglichkeiten (5). Die Option der Teilhabe an politischen Meinungsbildungs- und Gestaltungsprozessen erscheint unabdingbar zur Sicherstellung politischer Stabilität auf lokalem und nationalem Niveau. 


\section{Die sozialistische Urbanisierung und ihre Folgen für Jugoslawien und Belgrad}

Die gegenwärtige Problemkonstellation informeller Siedlungen in Post-Jugoslawien, Serbien und Belgrad geht ursprünglich auf die 1960er Jahre zurück. Die Ausbreitung des unrechtmäßigen Wohnungsbaus hat eine ganze Reihe von Ursachen (ILIć et al. 2004; Spevec \& Klempić Bogadi 2009, S. 458; Tsenkova 2010, S. 74; Zegarac 1999, S. 366). Ganz zentral sind zwei ineinander übergehende Entwicklungsstränge: Das ist erstens der im Falle Serbiens verzögerte, chaotische und sozial unverantwortliche Transformationsverlauf (TIOsAVLević 2011; VuJosević et al. 2010) und zweitens ganz generell das sozialistische Modell der Urbanisierung (Sмітн 1996).

Viele sozialistische Staaten verharrten nach dem Zweiten Weltkrieg in einem Stadium der „Under-urbanisation“ (KonRAD \& SzELENYI 1974, S. 92). Sie kennzeichnet Länder, in denen der Grad der Verstädterung - verglichen mit (westlichen) Ländern ähnlicher wirtschaftlicher Entwicklung - hinter dem Niveau der Industrialisierung zurückgeblieben war (PICKVANCE 2002). Under-urbanisation ist in dieser Argumentation eine Folge des Strebens nach hohem industriellem Wachstum mit der politischen Zielvorgabe, zur kapitalistischen Welt aufzuschließen. So hatten investive Ausgaben in den produktiven Sektor absoluten Vorrang (Murray \& Szelenyi 1984, S. 98ff.; Sailer-Fliege 1999, S. 8; Kostinskiy 2001, S. 452; Pickvance 2002). Das Diktum von der staatlich geförderten Industrialisierung, dem auch Jugoslawien folgte, ging in der politischen Ökonomie des Sozialismus erst zeitversetzt in eine Phase intensiver sozialistischer Verstädterung über (MurRay \& SzELENYI 1984, S. 100). Bis zur Systemtransformation führte das zu einem massiven Wachstum der städtischen Bevölkerung (ENYEDI 1992). Mit Ausnahme von Albanien war in den ehemals sozialistischen Ländern der Anteil der städtischen Bevölkerung nun wesentlich höher als in anderen Ländern mit ähnlich hohem BSP pro Kopf (BUCKLEY \& MinI 2000). Die konsequente Umsetzung jenes, priority approaches' mit begrenzten Ressourcen bedeutete letztlich die Minimierung der Ausgaben in nicht-produktiven Sektoren (Kornai 1992; Ofer 1976; Szelenyi 1996; SÝKora 2009, S. 388; Leetma et al. 2009, S. 439). Betroffen waren vor allem Investitionen in Einrichtungen der öffentlichen Daseinsvorsorge (Bildung, Gesundheit, Kultur, etc.) oder den Wohnungssektor, was in vielen Teilen des ehemaligen Jugoslawiens bis heute spürbar ist.

Auch dort überwog um die Mitte des 20. Jhs. noch der Anteil der ländlichen Bevölkerung. So lag es nahe, den Arbeitskräftebedarf für die beschleunigte Industrialisierung vor allem durch gering qualifizierte Erwerbspersonen aus den Dörfern zu decken. Das anfänglich massenhafte (Fern-)Pendeln ging dann rasch in das Umsiedeln in den städtischen Raum oder dessen Nähe über (vgl. Büschenfeld 1981, S. 104): Im Zeitraum von 1948 bis 1981 sind in Jugoslawien etwa 6,5 Mio. Menschen vom Land in die Stadt gezogen, was im europäischen Maßstab eine extreme Abwanderung darstellt (Vujović 1995; Petovar 2003a, b). Der Verstädterungsgrad in Serbien beispielsweise stieg von $17 \%$ (1948) auf 56\% (2002). 
Im Verlauf dieser Wachstumsphase blieb die jugoslawische Wohnbauförderung schwerpunkthaft auf die Städte konzentriert. Gleiches gilt für die Verstaatlichung von Grund und Boden. Der Wohnungsbestand in größeren Städten, und vor allem Immobilien mit mehreren Wohneinheiten wurden im Regelfall in gesellschaftliches Eigentum umgewandelt und so aus dem Marktgeschehen genommen (a.a.O.). Von der staatlichen Kontrolle des städtischen Wohnungsmarktes erwartete man sich vor allem ein sinkendes Mietpreisniveau, schließlich war gesellschaftliches Eigentum eines der wichtigsten Mittel, mit denen der Staat versuchte, befriedigende und relativ gleiche Wohnbedingungen für alle Einwohner vorzuhalten (Sмітн 1996). Auf der anderen Seite musste der Anspruch der Egalität mit Erfordernissen der Effektivität kombiniert werden, sodass von Investitionen in flächendeckende Infrastrukturen abgesehen wurde (Vujović 1995).

Der Mangel an Wohnraum in einigen Städten Jugoslawiens wurde schon gleich nach Einführung des Sozialismus offensichtlich, mancherorts (wie in Belgrad) noch verschärft durch die Kriegszerstörungen. So entwickelte sich die Zuteilung einer Wohnung in den Städten in der Folge, analog zur anderswo üblichen Praxis (SÝKORA 2009, S. 390), zu einer Art Belohnungssystem: Sie erfolgte häufig aufgrund ,gesellschaftlicher Verdienste“, d.h. Personen, die ,wichtige“ Aufgaben und Positionen besetzten, hatten Vorrang. Das waren vor allem Regierungskreise und solche Arbeiter, deren Tätigkeit vom Staat als höherwertig angesehen wurde. Diese profitierten dann in zweierlei Hinsicht: Sie bekamen neben dem ohnehin höheren Einkommen größere, qualitativ bessere und damit auch höher subventionierte Wohnungen; paradoxerweise bekamen also die Reichsten die höchsten staatlichen Zuwendungen (SZELENYI 1983; Smith 1996; Sailer-Fliege 1999, S. 8).

Im Gegensatz dazu musste eine große Zahl gering qualifizierter Arbeiter ihr Wohnungsproblem eigenständig lösen. Da privatwirtschaftliche, marktorientierte Bautätigkeit unterbunden war, fehlte ein geregelter privater oder öffentlicher Mietwohnungsmarkt. Ähnliches gilt für planerisch und infrastrukturell vorbereiteten Baugrund für den individuellen Wohnungsbau. Zugleich gestalteten sich die Verfahren zum Erlangen einer Baugenehmigung kompliziert, langwierig und kostenintensiv. So waren weniger privilegierte Gruppen quasi gezwungen, sich Häuser informell und bisweilen illegal auf freien Flächen, zumeist am Rand der Städte, zu bauen. Aus diesem Grund umgibt Belgrad, ebenso wie andere Städte in Serbien und Post-Jugoslawien, neben den ehemals staatlichen und betrieblichen Großwohnsiedlungen eine wenig urbanisierte städtische Peripherie, die zum großen Teil aus informell errichteten, privaten Familienwohnhäusern mit mangelhaftem Standard besteht (Vujović 1986). Physiognomisch ähneln diese Viertel (wie Kaluđerica) durchaus suburbanen Wohngürteln westeuropäischen Musters. Planerisch sind sie jedoch Ausdruck des laissez-faire im Sozialismus und der „period of degradation“ von 1988 bis 2000 mit ihrer (unfreiwilligen) Deregulierung (DJordjević \& DABović 2010, S. 301). Ursächlich sind sie allerdings weniger ein lebenszyklisch erklärbares Wohlstandsphänomen, sondern vice versa ein resilienter Anpassungsmechanismus mit Merkmalen sozialräumlicher Marginalität. 


\section{Der Wohnungsmarkt im Transformationskontext}

Die skizzierte Handlungsroutine deutet letztlich auf systemimmanente Verwertungsschwächen hin, die offensichtlich auch im jugoslawischen Sonderweg des Selbstverwaltungs-Sozialismus auftraten. Diese Einschätzung lässt sich ohne weiteres auf die post-jugoslawische Zeit übertragen, welche einen speziellen gesellschaftlichen und volkswirtschaftlichen Handlungsrahmen definiert. So waren die 1990er Jahre von den Zerfallskriegen und deren Folgen bestimmt. Die wirtschaftliche Entwicklung Serbiens wurde in jener Dekade mehrfach unterbrochen. Es kam zu einem Rückgang des Sozialproduktes, zu massiven Verschiebungen in der Wirtschaftsstruktur (insbesondere bemerkbar im Rückgang der Industrie), zum Rückgang von Investitionen und Beschäftigung sowie zum Anstieg der Arbeitslosenquote und letztlich zu einer Verarmung breiter Bevölkerungsschichten (UZB 2002; GRČIĆ \& RATKAJ, 2006; GöLER et al. 2007). Erst nach Eintritt ins neue Jahrtausend folgte eine Phase der wirtschaftlichen Erholung, die in der Regel durch die Dynamik kleiner und mittlerer Unternehmen getragen wurde und von schrittweisen strukturellen Transformationen vor allem im Bereich des Eigentums flankiert waren.

Die große Zahl an Wohnungen in staatlichem Eigentum stellte nach dem Niedergang des Sozialismus tatsächlich eines der wichtigsten Handlungsfelder dar (STRUYK 1996). Nun wurde Privateigentum gegenüber Staatseigentum als effektiver eingeschätzt; die niedrigen Mieten im staatlichen Segment behinderten die Entwicklung eines Immobilienmarktes. Die Privatisierung war also naheliegend. Sie wurde in Serbien durch die Hyperinflation Anfang der 1990er Jahre noch beschleunigt, denn unter diesen Bedingungen wurden Immobilien als wertbeständige Anlageform verstärkt nachgefragt. So befinden sich im administrativen Stadtgebiet Belgrads nunmehr fast alle Wohnungen in privatem Besitz (97,5\%; RZS 2002).

Faktisch führte die Privatisierung in Serbien zum Ausverkauf des gesellschaftlichen Vermögens, denn vor allem Bewohner, die in sozialistischer Zeit das Wohnrecht zugeteilt bekommen hatten, wurden damit zu Wohnungseigentümern. Wie bereits erwähnt, waren durch die Vergabepraxis vor allem Angehörige höherer gesellschaftlicher Schichten bevorzugt worden. Im Rahmen der Privatisierung konnten sie nun die relativ hochwertigen Wohnungen, die sie lange Zeit für geringe Mieten bewohnt hatten, erwerben. Angehörige niedrigerer sozialer Schichten dagegen bekamen, wie ausgeführt, seltener eine staatliche Wohnung (VuJović 1991). Falls doch, so war sie in der Regel von geringerer Qualität und wurde im Rahmen der Privatisierung von den Bewohnern selten erworben. So ist der nicht privatisierte Teil des Wohnungsbestandes heute vor allem Wohnraum mit unterdurchschnittlichem Standard. Deren Bewohner sind nun natürlich höchst verwundbar gegenüber marktwirtschaftlich bedingten Verdrängungsmechanismen - wie beginnende Gentrifizierungsprozesse in attraktiven Innenstadtlagen in den Bezirken Stari Grad und Vračar zeigen (TodoRIĆ \& RATKAJ 2011). Wohnungseigentümer, zu denen die Bewohner im Geschoßwohnungsbau genauso gehören wie jene in informellen Siedlungen, sind davon momentan noch weniger betroffen. 
Der Übergang Serbiens vom Sozialismus zum Kapitalismus sollte durch entsprechende Richtlinien sozialverträglich gestaltet werden; negative Konsequenzen, welche die ohnehin schon benachteiligten Einwohner treffen würden, sollten vermieden werden (Kovács 1999, S. 4; Petovar 2003a). In diesem Zusammenhang sollte neben privaten Freiheiten jedem Einzelnen das „Recht auf Bautätigkeit“ und „Recht auf Wohnung“ zugestanden werden, sodass Forderungen nach sozialer Gerechtigkeit mit Forderungen wirtschaftlicher Rationalität in Einklang gebracht werden können (VuJovıć 1995). Das ist bislang sicherlich nur bedingt gelungen, denn im Verlauf der Transformation konnten weder in Serbien noch in Belgrad die Probleme im Wohnungssektor gelöst werden.

Eine andere Folge der Zerfallskriege stellen Flüchtlinge aus den (ehemaligen) Teilrepubliken nach Serbien dar. Dazu kommt vor allem seit 1999 der Zustrom serbischer Bevölkerung aus dem Gebiet des Kosovo. Sie definieren in den Städten und ganz besonders in Belgrad kurzfristig eine große Nachfrage nach Wohnraum. Auch deshalb flossen in den Zeiten der Wirtschaftskrise private Investitionen vorzugsweise in informelle Bautätigkeiten, mit denen sich - häufig allerdings illegal - wirtschaftliche Gewinne erzielen ließen.

Dagegen fehlte es den serbischen Kommunen wegen der mangelhaften finanziellen Ausstattung und den unzureichenden Strategien für eine staatliche Wohnungspolitik an städtebaulichen Planungsgrundlagen. So konnte den mannigfaltigen sozioökonomischen Transformationseffekten kaum wirksam begegnet und die Öffnung der Märkte entsprechend begleitet werden. Das ist gleichzeitig ein Zeichen für die Schwäche von Politik und Staat (GöLER \& LeHMEIER 2011a, S. 38). Jener hatte die unrechtmäßigen Bautätigkeiten vor allem in den Krisenzeiten der 1990er Jahre, gleichsam im Sinne einer informellen Sozialpolitik, weiterhin toleriert und den Weg in eine in dieser Hinsicht hochgradig pfadabhängige Transformation geebnet.

\section{Informelles Wohnen in Belgrad}

Die Metropole Belgrad steht also schon seit Längerem im Brennpunkt der Problematik und stellt somit ein geeignetes Laboratorium für eine exemplarische Betrachtung dar. Bis zur Mitte der 1980er Jahre konzentrierte sich der informelle Wohnungsbau - ebenso wie in anderen jugoslawischen Stadtregionen - vor allem auf die randstädtischen Bereiche, also auf vorher nicht bebaute und zumeist landwirtschaftlich genutzte Areale außerhalb des verdichteten Stadtgebietes. Planerisch wurde dem Problem dadurch begegnet, dass in jeder Novellierung des Belgrader Stadtentwicklungsplans die Begrenzung des bebauten Gebietes einfach weiter gefasst wurde und die bereits existierenden informellen Siedlungen jeweils ex post in das Stadtgebiet einbezogen wurden. Mit der Verabschiedung des derzeit gültigen Masterplans (mit der Perspektive bis 2021) wurden erstmals ex ante Gebiete mit laufenden informellen Bautätigkeiten, welche bis dato von offizieller Seite ignoriert wurden, in das beplante Territorium einbezogen. Insgesamt handelt es sich um 52 informelle Siedlungsbereiche, die im Jahr 2002 etwa 43\% der gesamten Fläche mit Wohnnutzung ausmachten (UZB 2002). Dort befinden sich fast 43.000 Wohnungen mit offiziell gut 124.000 Einwohnern. 18 der 
informellen Siedlungen werden als unsicher oder sogar gefährlich für die Wohnnutzung eingestuft. Mehr als 20\% sind Siedlungen mit (Roma-)Bevölkerung, die in extremer Armut lebt (UN-HABITAT 2006, S. 17ff.).

Im Zeitraum von 1948 bis 2011 hat sich die Gesamtbevölkerung Belgrads weit mehr als verdoppelt (1.639.000 gegenüber gut 630.000 Einwohnern). Im engeren Stadtgebiet hat sie sich sogar nahezu verdreifacht (von 398.000 auf 1.136.000 Einwohner). Dort betrug die Zunahme allein zwischen 1971 und 1981 schon 21\%, und in den peripheren Teilen Belgrads sogar 23,2\%. Danach verlangsamte sich das Wachstum wieder etwas. Der Zuwachs von 2\% zwischen den beiden Volkszählungen von 1991 und 2002 entfiel speziell auf die Randbereiche der Agglomeration und dort unter anderem auf die Kommune Grocka, zu der Kaluđerica gehört. Jüngste Statistiken weisen bis 2011 wieder ein Plus von 4\% aus (64.000 Einwohner; RZS 2002 und 2011).

\section{Das Exempel Kaluđerica - Informalität als Marginalität?}

Die Bezeichnung „,informell“ für Siedlungen wie Kaluđerica scheint angemessen, denn die Wohnobjekte dort verfügen fast immer über legale Elemente: Das kann das Eigentum an Grund und Boden oder einzelne planerische Vorgaben betreffen, selbst wenn es aufgrund des improvisierten Baus oft an wesentlicher kommunaler Infrastruktur (Strom- und Wasserversorgung, Abwassersystem etc.) mangelt. Die Siedlung besteht aus einzeln stehenden, bisweilen dicht aneinander gebauten und in der Regel dreistöckigen Häusern oft respektabler Größe (vgl. Abb. 3). Sie finden sich auf zwar ungeplant und unrechtmäßig, aber regelhaft parzellierten ehemaligen Landwirtschaftsflächen; selten handelt es sich um offiziell ausgewiesenes Bauland. Sie bilden einen deutlichen Kontrast zu der sonst üblichen städtischen Blockbebauung aus jugoslawischer Zeit.

Administrativ gehört Kaluđerica zur etwa $10 \mathrm{~km}$ östlich stadtauswärts gelegenen Belgrader Vorstadtgemeinde Grocka. In Folge seiner Bevölkerungsdynamik und aufgrund des damit verbundenen intensiven ungeplanten Wohnungsbaus zwischen der Mitte der 1970er und dem Beginn der 1990er Jahre - in einer ersten Phase intensiver und informeller randstädtischer Entwicklung also (VoJković \& Devedžıć 2010, S. 104f.) - entwickelte sich Kaluđerica zur größten informellen Siedlung Serbiens und zu einer der größten dieser Art auf dem Balkan. Hinsichtlich der räumlichen Entwicklung Kaluđericas lassen sich dabei charakteristische Etappen unterscheiden (vgl. Abb. 4).

\section{Die Siedlung ...}

Die Siedlungsgenese geht auf die Mitte des 18. Jhs. zurück. Jener ältere Bereich ist, mit einer kleinen Grundschule und dem Gebäude der Ortsverwaltung, bis heute das gesellschaftliche Zentrum (Jovanović 1978). Im 19. und beginnenden 20. Jh. (2. Phase; vgl. Abb. 4) brachte der Zuwachs der ansässigen Familien sowie Zuwanderung eine Erweiterung mit sich; ähnlich war das während der Zwischenkriegszeit. Die 1950er und 1960er Jahre bedeuteten insbesondere eine Verdichtung des bebauten Areals, vor allem durch Haushaltsgründungen nachfolgender Generationen aus autochthonen Familien 


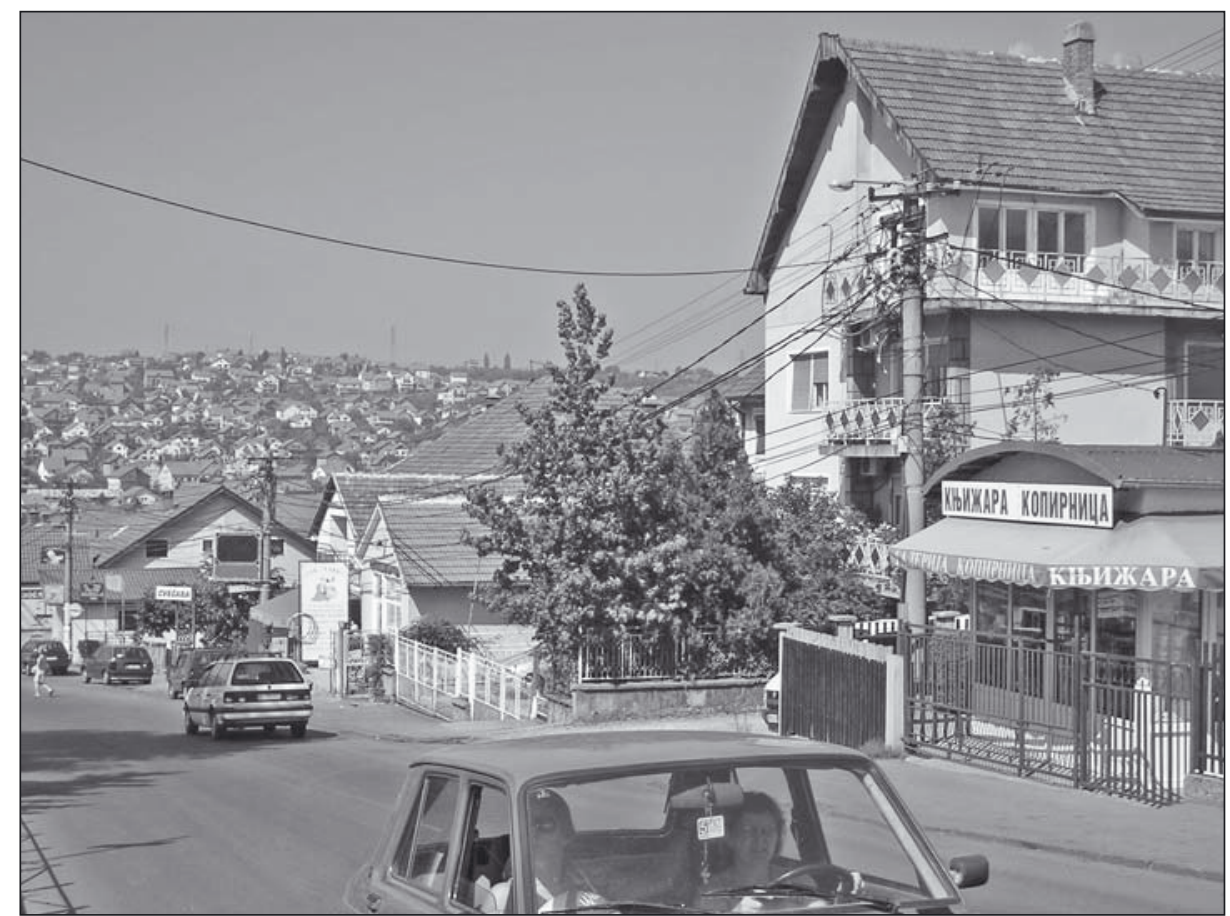

Abb. 3: Teil der informellen Siedlung Kaluđerica (Aufnahme D. GöLER 2008)

(Phase 3). Zum Ende der 1960er bzw. bis zur Mitte der 1970er Jahre lassen sich zwei unterschiedliche Erweiterungsbereiche identifizieren. Das ist erstens ein Areal mit ungeplanten Bautätigkeiten im Anschluss an zuvor geplant aufgesiedelte Bereiche (4a) sowie eine in den Jahren 1972/73 zur formalen Besiedlung vorgesehene Zone an der nördlichen Erschließungsstraße (4b), welche seitdem von einer öffentlichen Buslinie bedient wird (vgl. Abb. 5). Die Zeit von der Mitte der 1970er bis zum Anfang der 1990er Jahre (5. Phase) ist dann von explosionsartigen informellen Bautätigkeiten und einer entsprechend ausgreifenden Flächenausdehnung charakterisiert. 47\% des 2002 erfassten Gebäudebestandes stammte aus der Zeit von 1971 bis 1980 und weitere $31 \%$ aus dem Abschnitt 1981 bis 1990 (RZS 2002).

Auch die 1990er Jahre sind von einem erheblichen Flächenwachstum geprägt (Phase $6 ; 11 \%$ der Gebäude, s.o.). Rege informelle Bautätigkeiten setzten - neben einem kleineren Bereich im westlichen Kaluđerica - vor allem entlang einer der östlichen Belgrader Ausfallstraßen ein. Grund und Boden an diesem Abschnitt waren wegen der landschaftlich attraktiven Lage und seines hoch eingeschätzten Wertes bis dahin von jeglicher Bebauung frei gehalten worden. Die informelle räumliche und funktionale Expansion wurde dort durch den Zustrom von Flüchtlingen aus ex-jugoslawischen Republiken verstärkt. Wegen der politischen und ökonomischen Krise und mit Blick auf den sozialen Frieden musste der Staat diese Entwicklung tolerieren. 


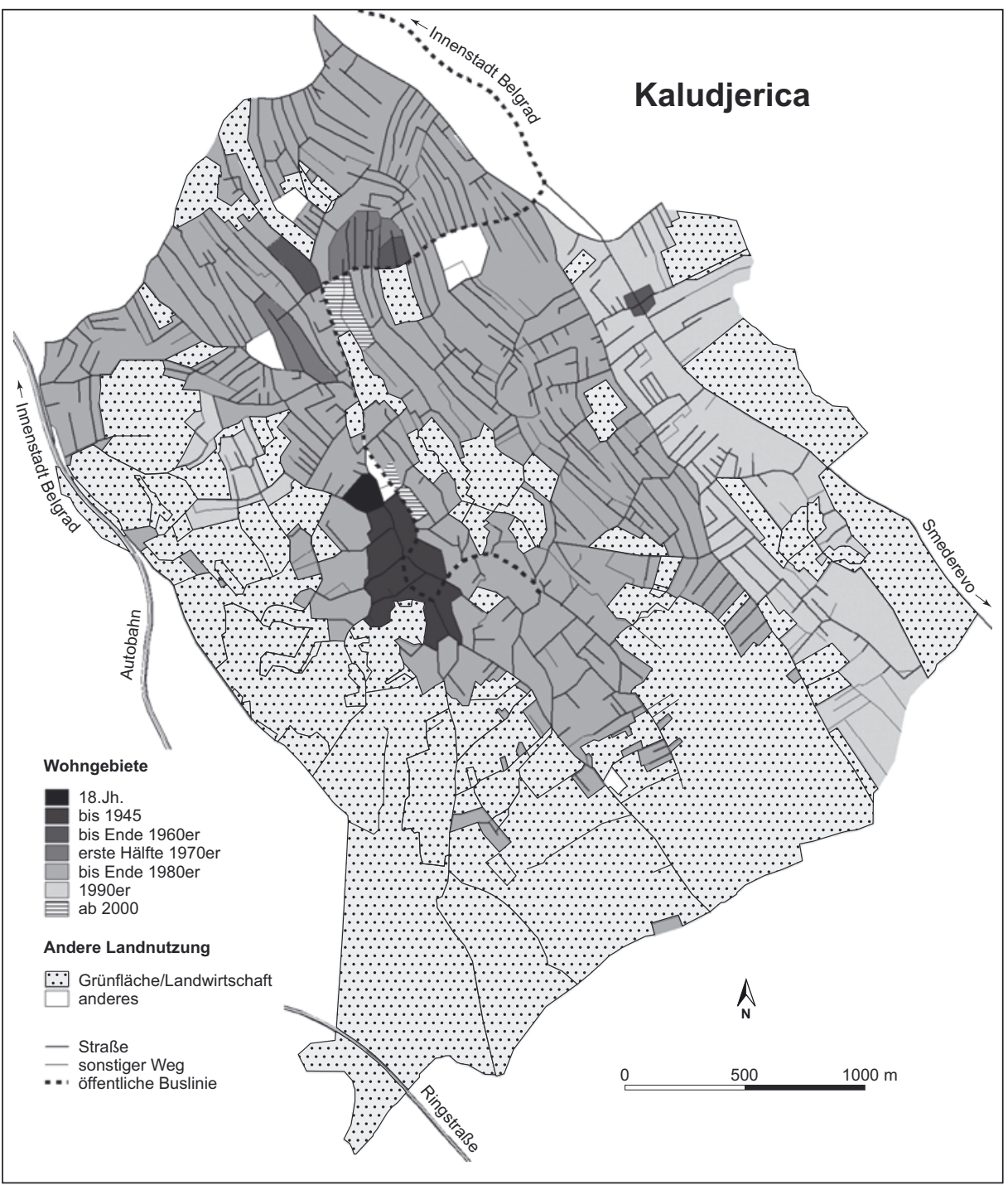

Quelle: Jovanović 1978 sowie Feldstudien der Autoren 2010/11

Abb. 4: Phasen der Siedlungsentwicklung Kaluđericas

Der Planungskrise folgte nach 2000 gleichsam die Wiederentdeckung strategischer Planung (DuordJević \& Dabović 2010, S. 301). So ist in Kaluđerica's siebenter Entwicklungsphase ein erster Einfluss der demokratischen Reformen erkennbar. Zwar konnten die informellen Tendenzen, trotz stärkeren Engagements der städtischen Behörden, nicht völlig unterbunden werden. Erste Erfolge entsprechender Bemühungen zeigen sich jedoch bei der laufenden Legalisierung des älteren Gebäudebestandes sowie bei 


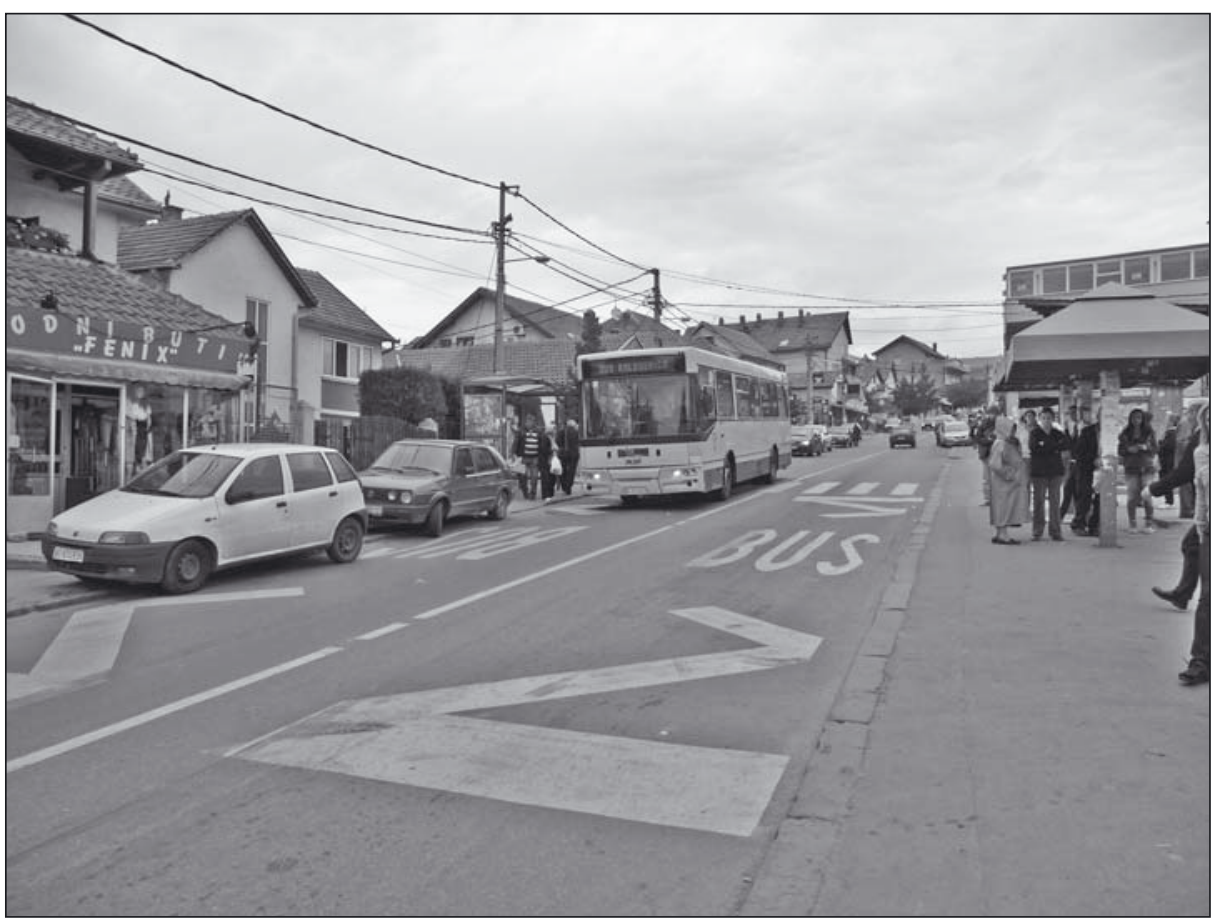

Abb. 5: Bushaltestelle an der zentralen Erschließungsstraße in Kaluđerica (Aufnahme D. GÖLER 2010)

der verstärkten Kontrolle derzeitiger Bautätigkeiten. Ziel ist es, informelle Aktivitäten künftig vollständig zu verhindern.

Konsequenterweise wurden zuletzt zwei neue Baugebiete formal ausgewiesen, beide entlang der öffentlichen Buslinie. Im ersten, im nördlichen Teil Kaluđericas, sind gemischte Gebäudestrukturen mit Einfamilienhäusern und Appartementgebäuden, im zweiten unweit des alten Ortskerns dagegen Appartementblocks vorgesehen. Diese Aktivitäten sind zweifelsohne zu begrüßen, gleichwohl - auch Anwohner äußern das - unverständlich bleibt, weshalb in dem Gebiet, das nach wie vor über kein Kanalsystem verfügt und in dem die Abwasserentsorgung gewöhnlich über Klärgruben erfolgt, Mehrfamilienhäuser gebaut werden. Bei Feldstudien im Februar 2011 konnte festgestellt werden, dass dort einige Wohnungen nicht verkauft waren, obwohl die annoncierten Preise mit 800 bis $900 €$ je $\mathrm{m}^{2}$ deutlich unter dem Niveau infrastrukturell angebundener und dem Entwicklungsplan entsprechender Appartements im suburbanen Raum Belgrads liegen.

\section{... und ihre Gesellschaft}

Die progressive Siedlungsentwicklung Kaluđericas ist durch die kontinuierliche Zunahme der Einwohnerzahl mit einem Schwerpunkt während der 1970er Jahre, der Zeit 


\begin{tabular}{|c|c|c|}
\hline Jahr & Einwohnerzahl & Wachstumsindex \\
\hline 1844 & 227 & 249,8 \\
\hline 1900 & 567 & 164,7 \\
\hline 1948 & 934 & 108,2 \\
\hline 1953 & 1.011 & 105,4 \\
\hline 1961 & 1.066 & 179,1 \\
\hline 1971 & 1.909 & 651,4 \\
\hline 1981 & 12.435 & 147,3 \\
\hline 1991 & 18.326 & 121,4 \\
\hline 2002 & 22.248 & 119,3 \\
\hline 2011 & 26.549 & \\
\hline
\end{tabular}

Quelle: RZS 2002; RZS 2011

Tab. 1: Bevölkerungsentwicklung Kaluđericas von 1844 bis 2011

mit der stärksten ,,wilden“ Bautätigkeit, bedingt. Allein in dieser Dekade (1971-1981) nahm die Bevölkerung um das 6,5-Fache zu (vgl. Tab. 1). Seitdem verlangsamte sich die Intensität des Wachstums. Die Einwohnerzahl beläuft sich gemäß der letzten Zählung auf 26.549 Einwohner in 8.831 Haushalten (RZS 2011). Die tatsächliche Zahl dürfte wesentlich höher liegen, auch wenn die Angabe von 70.000 (bei BoBIć 2004, S. 45) für Kaluđerica allein zu hoch gegriffen scheint. Im Rahmen unserer Feldstudien wurde eine Größenordnung von 50.000 bis 60.000 Einwohnern genannt (ähnlich bei Tsenkova 2010, S. 76). An Ort und Stelle geht man also von zumindest dem Doppelten der offiziellen Einwohnerzahl aus.

Die erhebliche Abweichung zum offiziellen Zensus hat mehrere Gründe. So sind selbst langjährige Mieter und vor allem Untermieter nicht gemeldet, da die Eigentümer der Häuser als Vermieter sonst Steuern entrichten müssten. Darüber hinaus sind selbst viele Hauseigentümer nicht registriert. Das hängt mit früheren Gepflogenheiten zusammen, als Wohnungen nach Dringlichkeit an Wohnungslose vergeben wurden. Obwohl sie nun bereits ein Haus gebaut haben, setzen vor allem die zahlreichen zugezogenen Flüchtlinge die Tradition der Nicht-Registrierung fort, da sie nach wie vor vom Staat erwarten, ihr Wohnungsproblem gelöst zu bekommen.

Vor allem die Zahl der Zuwanderer hat seit den 1970ern kontinuierlich zugenommen (RZS 2002). Etwa ein Viertel der Bewohner (5.621) ist erst zwischen 1991 und 2002 zugezogen. Darunter befinden sich Personen aus Belgrad (Allerdings liegt deren Anteil nur bei knapp $12 \%$.) sowie aus Kroatien $(6,8 \%)$ und Bosnien und Herzegowina $(3,1 \%)$. Zu den üblichen sozio-ökonomischen Gründen für die Suburbanisierung tritt also hier der Zerfall Jugoslawiens als Zuwanderungsgrund.

Die Erwerbsquote der (registrierten) Einwohner Kaluđericas liegt bei 52,6\% (Stand 2002) und damit etwas über dem Durchschnittswert für das Gebiet des Masterplans Belgrads (50,8\%). Der größte Anteil der Beschäftigung entfällt auf den tertiären (37\%) 
und sekundären Sektor (31\%; vgl. Tab. 2). Im quartären Sektor sind es 29\%, was deutlich unter dem Belgrader Durchschnitt liegt. Die Beschäftigung in höherwertigen Dienstleistungen scheint unter- und in geringwertigeren stark überrepräsentiert. Darin spiegelt sich das vergleichsweise niedrige Qualifikationsniveau der Einwohner wider. Der Anteil an Erwerbspersonen mit mangelhafter Ausbildung oder jener, die nur die vorgeschriebene Schulpflicht (oder weniger) erfüllt haben, liegt bei 26,1\% (Belgrad: $17,5 \%$ ). Ebenso beträgt beispielsweise der Anteil der über 24-Jährigen, die eine hohe Ausbildung haben, in Kaluđerica nur ca. 5\%, in Belgrad dagegen 19,3\%.

\begin{tabular}{|l|c|c|}
\hline Wirtschaftssektor in \% & Kaluđerica & Belgrad \\
\hline Primär & 2,2 & 1,6 \\
\hline Sekundär & 31,5 & 23,7 \\
\hline Tertiär & 37,0 & 31,8 \\
\hline Quartär & 29,0 & 41,1 \\
\hline Unbekannt & 0,4 & 1,8 \\
\hline
\end{tabular}

Quelle: RZS 2002

Tab. 2: Anteil der Beschäftigten in Kaluđerica und in Belgrad (Gebiet des Masterplans) 2002; Angaben in \%

Eine genauere Analyse der verfügbaren Daten zeigt weiterhin, dass 94,2\% der Beschäftigten ihrer Arbeit in Belgrad nachgehen, die weit überwiegende Mehrheit außerhalb von Kaluđerica; d.h. 86,5\% der Beschäftigten sind Pendler. Sie arbeiten zum größten Teil in Grocka selbst (19,7\% der Beschäftigten), im benachbarten Zvezdara $(17,9 \%)$ oder im zentralen Belgrader Bezirk Stari Grad (12,7\%). Aufgrund der randlichen Lage und der zurückzulegenden großen Distanzen, z.B. nach Stari Grad, müssen die Beschäftigten aus Kaluđerica deutlich mehr Zeit auf dem Weg zum Arbeitsplatz aufwenden als es in Belgrad üblich ist (ca. 38 Minuten gegenüber 27,5 Minuten). Dieser zusätzliche Mobilitätsaufwand ist ein Indikator für die vergleichsweise geringere Effizienz der räumlichen Organisation der Siedlung (RATKaJ 2009).

Knapp 20\% der Bevölkerung Kaluđericas sind schulpflichtige Kinder und Jugendliche. Fast alle besuchen eine Bildungseinrichtung in der Munizipalität Belgrad, $60 \%$ davon außerhalb des Wohnortes Kaluđerica. Bis zur achten Klasse werden in der Regel wohnortnahe Schulen besucht (gut drei Viertel in Kaluđerica, daneben im benachbarten Zvezdara [14,2\%], Grocka [3,4\%] und Vinča [3\%]). Bei den weiterführenden Schulen ist der Anteil täglicher Bildungspendler mit mehr als $90 \%$ sehr hoch.

\section{Chancen und Risiken des Lebens in der Informalität}

Das Leben in der Informalität in Kaluđerica scheint den Bewohnern durchaus Vorteile zu bieten. Da ist zunächst die verkehrsgünstige Lage in der Nähe der Umgehungsstraße und der Autobahn, welche eine gute Anbindung und Erreichbarkeit vieler Einrichtungen mit zentralörtlichen Funktionen (Arbeit, Gesundheit, Bildung 
etc.), die vorwiegend in Belgrad angeboten und nachgefragt werden, gewährleistet. In Kaluđerica selbst gibt es einige öffentliche Einrichtungen, so ein Verwaltungsgebäude, eine Grundschule oder die medizinische Ambulanz. Die Versorgung mit Gütern des täglichen Bedarfs ist durch einen Obst- und Gemüsemarkt gewährleistet (vgl. Abb. 6). Insgesamt aber ist die Kapazität solcher Infrastrukturen mit Blick auf die große Zahl der Einwohner und deren Bedürfnisse sicherlich zu gering.

Die Grundstückspreise beispielsweise für landwirtschaftliche Nutzfläche in privatem Besitz (also potenzielles Bauland) sind hier mit durchschnittlich $25 € / \mathrm{m}^{2}$ nach wie vor relativ niedrig. Die Spannweite reicht von 11 bis $50 € / \mathrm{m}^{2}$, jeweils in Abhängigkeit von der Verfügbarkeit von Straßenanbindung, Wasser und Elektrizität (vgl. http://e-belgrade.net/). In diesem Zusammenhang berichten Bewohner vom „Vorteil““ durch die offenbar unzureichende Kommunikation zwischen der lokalen Verwaltung von Kaluđerica mit der übergeordneten Gemeinde Grocka: Das manifestiere sich unter anderem im Immobiliensektor durch die - im Vergleich mit dem benachbarten Zvezdara oder anderen Orten in vergleichbarer Entfernung zum Stadtzentrum - schwache Kontrolle der formellen und informellen Bautätigkeiten in Kaluđerica. Hinsichtlich der Problematik einer gewissen Ferne der Verwaltung und dem Wunsch einer Herauslösung aus der Gemeinde Grocka formieren sich unter den Bewohnern zwei Bewegungen. Die eine favorisiert den Anschluss an Zvezdara und die andere

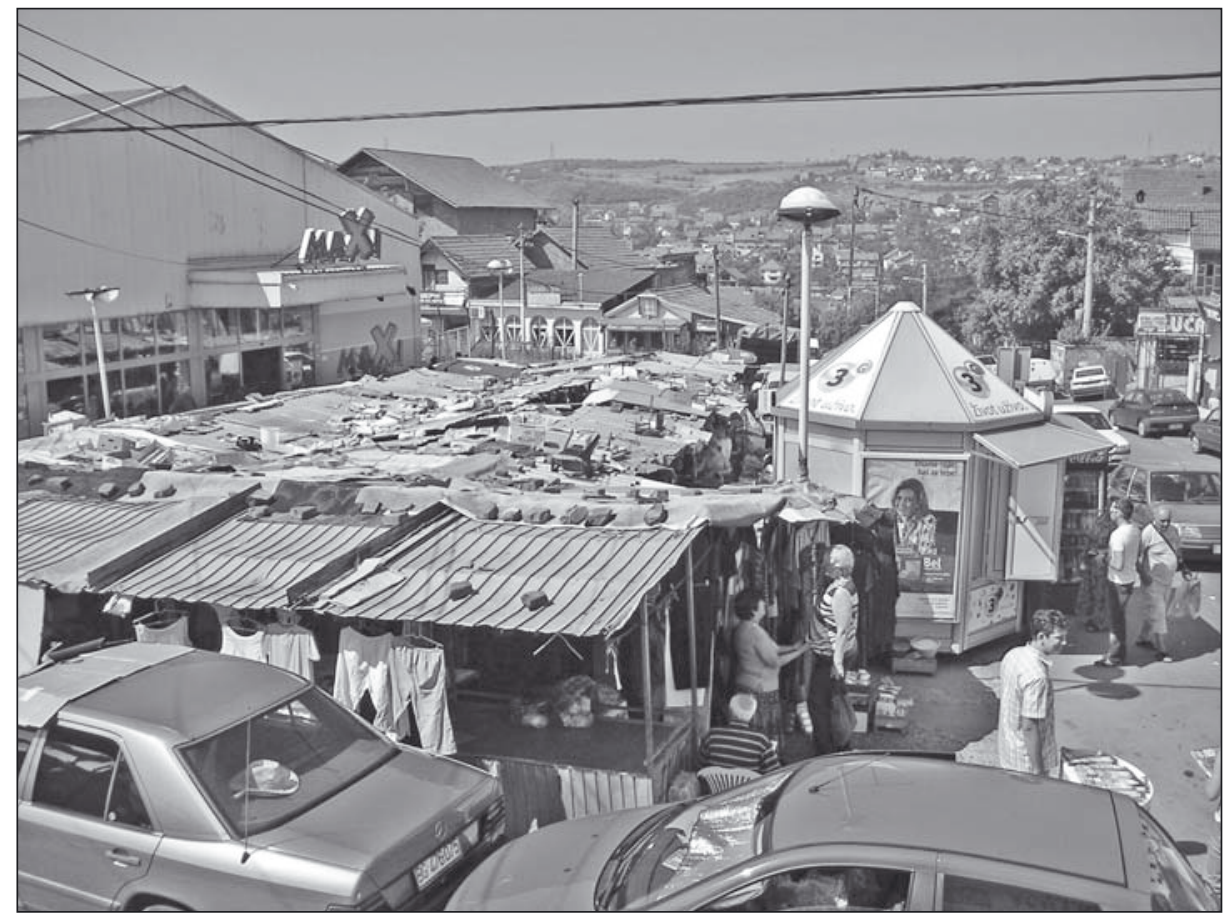

Abb. 6: Der zentrale Markt in Kaluđerica (Aufnahme D. Göler 2010); im Hintergrund Teile der informellen Siedlung 
die Bildung einer eigenständigen kommunalen Einheit gemeinsam mit vier anderen umliegenden Siedlungen (Vinča, Leštane, Boleč und Ritopek).

Der informelle Wohnungsbau hatte seinen Ursprung gewöhnlich darin, dass $\mathrm{Zu}$ wanderer und andere Interessenten landwirtschaftliche Parzellen erwarben und ohne behördliche Kenntnis und Genehmigung ein Gebäude errichteten. Maßnahmen, mit denen in Belgrad seit den 1960er Jahren unrechtmäßigen Bautätigkeiten eigentlich vorgebeugt werden sollte, wurden nur sporadisch, nie aber konsequent verfolgt. So wurden in der ersten Hälfte der 1970er Jahre, als das Gebiet von Kaluđerica in die Belgrader Stadtentwicklungsplanung (Masterplan) einbezogen wurde, seitens der Behörden Versuche unternommen, dort die Bau- und Siedlungstätigkeit zu kontrollieren. Vereinzelt wurden sogar informell errichtete Gebäude beseitigt. Ziele des Rückbaus waren allenfalls unfertige Objekte, welche bei Inspektionen als offensichtlich dauerhaft unbewohnt angetroffen wurden. Solche Kontrollen waren nicht wirklich implementiert, wurden relativ kurzfristig angesetzt und alles in allem wenig rigide durchgeführt. Ungeachtet dessen setzten nach dem Abriss umgehend, quasi über Nacht, auf dem Grundstück regelmäßig neuerliche Bautätigkeiten ein.

Im Rahmen der Feldstudien berichtet ein Interviewpartner zu diesem Procedere: „Als ich hörte, dass die Inspekteure kamen und begannen, Gebäude zu zerstören, war mein Haus nur teilweise fertiggestellt, zu diesem Zeitpunkt ohne Wasser, Elektrizität, Badezimmer und andere Notwendigkeiten, die man zum täglichen Leben benötigt. Trotzdem, ich zog in das Haus, zusammen mit meiner Frau und den kleinen Kindern. Das war die einzige Möglichkeit, den Leuten klar zu machen, dass wir keinen anderen Ort zum Wohnen hatten und dass sie unser Haus verschonen."

Viele der Belange einer funktionsräumlichen Organisation der Siedlung sind ungelöst, darunter Fragen eines adäquaten öffentlichen Transportsystems mit entsprechenden Kapazitäten, des hindernisfreien Zugangs für Krankenwagen, der Abfallentsorgung, der Zuständigkeit von Feuerwehren und anderer öffentlicher Dienstleistungen. Dazu ein anderer Befragter:

„Ja, wir haben eine Polizeiwache, ein neues medizinisches Zentrum, das jedoch noch nicht voll ausgestattet ist, drei Grundschulen, Kindertagesstätte, Bücherei, Einkaufsgelegenheiten, Bank, Post, gute öffentliche Verkehrsanbindung. [...] Aber den Stromanschluss und befestigte Straßen zu unseren Häusern haben wir selbst hergestellt. [...] Nur zwei Straßen haben Gehsteige; in anderen wird es schon schwierig, wenn sich zwei Fahrzeuge begegnen. Wenn jemand in der Straße parkt, dann wird es für einen Krankenwagen unmöglich, uns zu erreichen - gar nicht zu denken an die Feuerwehr oder einen Müllwagen. [...] Im Winter sind die Straßen mit Schnee bedeckt. Normalerweise wird der Müll, den wir in Plastiktüten im Hof lagern, zweimal die Woche abgeholt, natürlich nur, wenn kein Fahrzeug parkt oder Schnee liegt. Dann müssen wir bis zur nächsten Woche warten. Das Ganze ist noch schlimmer, seit unsere Straße eine Sackgasse ist, weil ein Nachbar sein Haus darauf gebaut hat ..." 


\section{Formalisierung und Legalisierung als Herausforderungen}

Die Herausforderung der nachträglichen Formalisierung, also einer Legalisierung und Aufwertung der informell und/oder illegal errichteten Objekte in Kaluđerica ist, wie in anderen ähnlichen Siedlungen, augenscheinlich. Diesbezügliche Ansätze haben eine genauso lange Tradition wie das informelle Wohnen selbst. Ein erster (erfolgloser) Versuch von Behörden, dem Problem vor allem der illegalen Bebauung in Belgrad durch Abriss zu begegnen, wurde schon 1961 unternommen (ZEGARAC 1999, S. 366). Weitere folgten nicht nur in Kaluđerica (s.o.), sodass auch hinsichtlich der Problemlösung „Routinen“ vermutet werden dürfen.

Allerdings wurden die Bemühungen zuletzt erheblich intensiviert. So wurde im Hinblick auf das Ziel der Legalisierung informell erbauter Objekte in Serbien 2003 das „Gesetz zu Planung und Bautätigkeiten“ erlassen (Službeni Glasnik Republike Srbije, br. 47/03). Es reduzierte die Vorschriften und die notwendigen Unterlagen für den Erhalt von Genehmigungen und setzte ein geringes Entgelt für die Legalisierung fest, welches den Stadtgemeinden zugesprochen wird. Allein in Belgrad wurden 130.000 Anträge auf Regulierung gestellt (UN-HABITAT 2006, S. 19). Der eher symbolische Anteil tatsächlich legalisierter Objekte (weniger als 3\%) spricht allerdings für ein fragwürdiges Procedere in der Ausführung des Gesetzes. Aus eben diesem Grund hat sich der Staat im Jahr 2009 erneut des Problems angenommen und eine neue Gesetzesgrundlage erlassen (Službeni Glasnik Republike Srbije, 72/2009 vom 11. September 2009), welche die Rechte der Eigentümer illegaler Objekte weiter stärkt.

Zum Zeitpunkt der Recherchen zeichnete sich jedoch bereits ab, dass mit der neuen Regulative nicht unbedingt bessere Ergebnisse erzielt werden, speziell in Kaluđerica. In den meisten Fällen liegt das an den ungelösten Fragen im Verhältnis zwischen Liegenschaft und Eigentum. Um in den Prozess der Legalisierung zu gelangen ist es notwendig, dass dem Eigentümer des informell erbauten Objektes gleichzeitig auch der Grund gehört. Zwar sind die meisten Antragsteller faktisch tatsächlich Eigentümer des Landes, auf dem ihr Haus steht. Nur können sie es im juristischen Sinn nicht nachweisen. Das liegt daran, dass die Zuwanderer gewöhnlich eine landwirtschaftliche Parzelle aus privatem Familienbesitz erworben und den entsprechenden Vertrag nur mit einem der Familienmitglieder abgeschlossen hatten. Jenes war formal möglicherweise gar nicht der Eigentümer, da im Kataster beispielsweise immer noch der Vater oder Großvater eingetragen war. Da eine große Zahl der Erblasser (die damaligen tatsächlichen Eigentümer) zwischenzeitlich verschieden ist, ist das Nachvollziehen des Eigentumsübertrages und die Lösung des Problems der Legalisierung schwierig. Und selbst in Fällen, in denen der Erblasser noch lebt, sind die Hauseigentümer in einer ungünstigen Verhandlungsposition. Theoretisch können sie für das gleiche Grundstück abermals zur Kasse gebeten werden, um dann das Placet zur Legalisierung zu erlangen.

Gerade zum Thema Legalisierung gibt es unterschiedliche Einstellungen zwischen den alteingesessenen Bewohnern und den erst in jüngerer Zeit Zugezogenen. Alle gemeinsam stimmen jedoch überein in der Feststellung: „Wer eines Tages nach Kaluđerica kommt, findet niemals wieder heraus“. Es verbleibt die Frage, ob das als Fluch oder als Segen zu verstehen ist. 


\section{Fazit}

Die Analysen zum Problem informeller Siedlungen in Jugoslawien und Serbien, die Ausführungen zur Problematik im sozioökonomischen Gravitationszentrum Belgrad und speziell im Mikrokosmos Kaluđerica haben deutlich gemacht, wie eng dort der gordische Knoten aus den Eckpfeilern städtebaulicher Informalität, sozialer Marginalität und tief verankerter lebensweltlicher Handlungsroutinen geknüpft ist. Es hängt mit der spezifischen Vorgeschichte zusammen, dass sich hier die anderswo im Transformationskontext üblichen marktgesteuerten Suburbanisierungsmuster (vgl. LeEtma, TAMmARU \& ANNISTE 2009) bislang nicht durchgesetzt haben. Zweifelsohne, Infomalität ist im gegebenen Zusammenhang nicht zwingend ein Armutsphänomen, genauso wenig wie Suburbanisierung hier nicht als Wohlstandsphänomen gedeutet werden kann. Marginalität resultiert im gezeigten Fall vor allem aus der Unsicherheit bezüglich der Rechtsverhältnisse der Immobilien, wenngleich diese Unsicherheit und das resultierende, potenziell sehr hohe Maß der Verwundbarkeit zur Normalität in Vierteln wie Kaluđerica gehört. Die Entstehung und die dauerhafte Präsenz des Phänomens informellen Siedelns kann insofern in der Tat als langfristig etablierte, im Sozialismus begonnene und im Post-Sozialismus weitergeführte Routine interpretiert werden. Sie hat alle Diskontinuitäten des zurückliegenden halben Jahrhunderts überdauert, ebenso wie alle bisherigen Versuche des Gegensteuerns. Es bleibt abzuwarten, wie sich das im Zusammenhang mit den Bemühungen der nachträglichen Legalisierung verhalten wird.

\section{Literaturverzeichnis}

Bobić M. (2004), Belgrad: Eine Multiple Edge City. In: StadtBauwelt, 163, S. 42-49.

Bouzarovski S. (2011), City Profile Skopje. In: Cities, 28, S. 265-277.

Bouzarovski S., Salukvadze J., Gentile M. (2011), A socially resilient urban transition? The contested landscapes of apartment building extensions in two post-communist cities. In: Urban Studies, 48, 13, S. 2689-2714.

Buckley R.M., Mini F. (2000), From Commissars to Mayors: Cities in the Transition Economies. Washington, D.C., The World Bank.

Büschenfeld H. (1981), Jugoslawien (= Länderprofile - Geographische Strukturen, Daten Entwicklungen). Stuttgart, Ernst Klett.

Djordjević D., DABOvić T. (2010), Strategic planning as instrument of spatial development of Belgrade. In: Krevs M., Duordjević D., Pichler-Milanović N. (Hrsg.), Challenges of spatial development of Ljubljana and Belgrade (= GeograFF, 8), S. 301-322. Ljubljana, Scientific Publishing House of the Faculty of Arts.

Doka Dh., Göler D. (2010), Vom Latecomer der Urbanisierung zum Newcomer im Postkommunismus - Tirana's Weg ins 21. Jahrhundert. In: Bohn Th., CALIC M.-J. (Hrsg.), Urbanisierung und Stadtentwicklung in Südosteuropa vom 19. bis zum 21. Jahrhundert (= Südosteuropa-Jahrbuch, 37), S. 307-317. München, Südosteuropa-Gesellschaft.

Economic Commission for Europe (Hrsg.) (2006), Country Profiles on the Housing Sector Serbia and Montenegro. New York - Geneva, United Nations.

ENYEDI G. (1992), Urbanization in east central Europe: social processes and societal responses in the state socialist systems. In: Urban Studies, 29, 6, S. 869-880. 
Fassmann H. (2007), Transformationsforschung in der Geographie. In: Gebhardt H., Glaser R., Radtke U., Reuber P. (Hrsg.), Geographie. Physische Geographie und Humangeographie, S. 672-673. Heidelberg, Springer.

GöLER D., GrČIĆ M., RATKAJ I. (2007), Tendenzen der jüngeren industriellen Entwicklung in Serbien und ihre regionale Differenzierung - untersucht mit einem quantitativen Analyseansatz. In: Mitteilungen der Österreichischen Geographischen Gesellschaft, 149, S. 109-132.

Göler D., Lehmeier H. (2011a), Balkanmetropolen. Urbane Entwicklungen in Belgrad, Bukarest und Sofia. In: Geographische Rundschau, 63, 4, S. 34-41.

Göler D., Lehmeier H. (2011b), Belgrad - Periphere Metropole im europäischen Städtesystem? In: Matznetter W., Musil R. (Hrsg.), Europas Städte in der Weltwirtschaft des 20. Jahrhunderts, S. 341-358. Wien, Mandelbaum.

Grabher G., Stark D. (1997), Organizing Diversity: Evolutionary Theory, Network Analysis, and Post-Socialism. In: Regional Studies, 31, 5, S. 533-544.

GRČIĆ M., RATKaJ I. (2006), Strukturne promene i regionalna diferencijacija industrije Srbije u periodu tranzicije (1988-2005) [Strukturwandel und regionale Differenzierung der serbischen Industrie in der Transformationsperiode (1988-2005)]. In: Glasnik Srpskog geografskog društva, 86, 2, S. 97-112.

Harris C.D. (1972), Cities of the Soviet Union: Studies in their Functions, Size, Density, and Growth. Chicago, Rand McNally.

Herrle P. (2010), Informelle Siedlungen. In: Henckel D., Kuczkowski K. von, Lau P., PahlWeber E., Stellmacher F. (Hrsg.), Planen - Bauen - Umwelt. Ein Handbuch, S. 232-234. Wiesbaden, VS Verlag für Sozialwissenschaften.

Hirt S. (2009), City Profile Belgrade, Serbia. In: Cities, 26, S. 293-303.

Hütteroth W.-D., HöHFeld V. (2002), Türkei. Geographie, Geschichte, Wirtschaft, Politik (= Wissenschaftliche Länderkunden). Darmstadt, Wissenschaftlicher Buchverlag.

Ilić V.A., Petovar K., Čolić R. (2004), Bespravna izgradnja u Srbiji: geneza i perspektive rešavanja problema [Illegales Bauen in Serbien: Entstehung und Perspektiven der Problemlösung]. In: Strateški okvir za održivi razvoj Srbije, Posebna izdanja, 44, S. 253-261. Beograd, Institut za arhitekturu i urbanizam Srbije.

Jovanović R. (1978), Preobražaj prigradskih naselja na primeru Sremčice i Kaluđerice [Die Transformation der Vorstädte am Beispiel Sremčica und Kaluđerica]. In: Zbornik radova Geografskog instituta „Jovan Cvijici“, SANU, 30, S. 145-195.

Konrad G., Szelenyi I. (1974), Social conflicts of under-urbanization. In: Brown A.A., Licari J.A., Neuberger E. (Hrsg.), Urban and Social Economics in Market and Planned Economies, 1, S. 206-226. New York, Praeger.

Kornai J. (1992), The Socialist System. The Political Economy of Communism. Princeton, Princeton University Press.

Kostinskiy G. (2001), Post-Socialist Cities in Flux. In: Paddison R. (Hrsg.), Handbook of Urban Studies, S. 451-465. London - New Delhi, Thousand Oaks.

Kovács Z. (1999), Cities from state-socialism to global capitalism: an introduction. In: GeoJournal, 49, 1, S. 1-6.

Leetma K., Tammaru T., Anniste K. (2009), From Priority-led to Market-led Suburbanisation in a post-communist Metropolis. In: Tijdschrift voor Economische en Sociale Geografie, 100,4 , S. 436-453.

Lewis R.A., Rowland R.H. (1979), Population Redistribution in the USSR: Its Impact on Society, 1897-1977. New York, Praeger.

Ministerial Conference on Informal Settlements in South Eastern Europe (Hrsg.) (2004), Vienna Declaration on Informal Settlements in South Eastern Europe, Vienna, $28^{\text {th }}$ Sep- 
tember $-1^{\text {st }}$ October 2004. - http://www.stabilitypact.org/humi/041001-conference.html (letztmals aufgerufen 20.09.2009).

Murray P., Szelenyi I. (1984), The City in the Transition to Socialism. In: International Journal of Urban and Regional Research, 8, S. 90-107.

OfER G. (1976), Industrial Structure, Urbanization and the Growth of Socialist Countries. In: Quarterly Journal of Economics, 90, S. 219-244.

Petovar K. (2003a), Socijalni razvoj i regionalne granice na primeru regiona Beograd [Soziale Entwicklung und regionale Grenzen am Beispiel der Region Belgrad]. In: STOJкоv U.B. (Hrsg.), Beograd i njegov region, S. 202-209. Beograd, Geografski fakultet.

Petovar K. (2003b), Naši gradovi između države i građanima - urbana sociologija [Unsere Städte zwischen Staat und Bürgern - eine Stadtsoziologie]. Beograd, Geografski fakultet Univerziteta u Beogradu.

Pickvance C. (2002), State Socialism, Post-Socialism and their urban Pattern: Theorizing the Central and Eastern European Experience. In: EAde J., Mele C. (Hrsg.), Understanding the City, S. 183-202. Oxford, Blackwell.

RATKAJ I. (2009), Prostorno-funkcionalna organizacija Beograda [Die räumlich-funktionale Organisation von Belgrad]. Beograd, Univerzitet u Beogradu - Geografski fakultet.

RepubličKi ZAVod ZA STATISTIKU (RZS) (Hrsg.) (2002), Popis stanovništva, domačinstava i stanova u 2002 [Bevölkerungs-, Gebäude- und Wohnungszählung von 2002]. Beograd.

RepubličKi Zavod za statistiku (RZS) (Hrsg.) (2011), 2011 Census of Population, Households and Dwellings in the Republic of Serbia - First results. - http://webrzs.stat.gov.rs/ WebSite/Public/PublicationView.asp $x$ ? $p$ Key $=41 \&$ pubType $=9$

SAILER-Fliege U. (1999), Characteristics of post-socialist urban transformation in East Central Europe. In: GeoJournal, 49, 1, S. 7-16.

Schulz M. (1991), Der Tauschwohnungsmarkt in der zentralistischen Planwirtschaft - Das Beispiel Ost-Berlin (= ISR-Forschungsberichte, 3). Wien, Verlag der Österreichischen Akademie der Wissenschaften.

Smith D.M. (1996), The Socialist City. In: Andrusz G., Harloe M., Szelenyi I. (Hrsg.), Cities after Socialism: Urban and Regional Change and Conflict in Post-Socialist Societies, S. 70-99. Oxford, Blackwell.

Spevec D., Klempić Bogadi S. (2009), Croatian Cities under Transformation: New Tendencies in Housing and Segregation. In: Tijdschrift voor Economische en Sociale Geografie, 100,4 , S. 451-468.

Stadelbauer J. (1991), Kolchozmärkte in der Sowjetunion. Geographische Studien zu Struktur, Tradition und Entwicklung des privaten Einzelhandels (= Mainzer Geographische Studien, 36). Mainz, Geographisches Institut der Johannes-Gutenberg-Universität Mainz.

Stark D. (1992), Path Dependency and Privatization Strategies in East Central Europe. In: Eastern European Politics and Societies, 6, S. 17-51.

STRUYK R. (1996), Economic restructuring of the Soviet Bloc: The case of housing. Washington D.C., The Urban Institute Press.

SÝ́ora L. (2008), Revolutionary Change, Evolutionary Adaption and New Path Dependencies: Socialism, Capitalism and Transformations in Urban Spatial Organizations. In: STRUBeLT W., Gorzelak G. (Hrsg.), City and region. Papers in Honour of Jiří Musil, S. 283-295. Opladen - Farmington Hills, Budrich UniPres.

SÝ́KORA L. (2009), Post-Socialist Cities. In: Kitchin R., Thrift N. (Hrsg.), International Encyclopedia of Human Geography, 8, S. 387-395. Amsterdam et al., Elsevier.

SÝ́ora L., Bouzarovski S. (2012), Multiple Transformations: Conceptualising the Postcommunist Urban Transition. In: Urban Studies, 49, 1, S. 43-60. 
SZELENYi I. (1983), Urban inequalities under state socialism. Oxford - New York, Oxford University Press.

Szelenyi I. (1996), Cities under Socialism - and after. In: Andrusz G., Harloe M., Szelenyi I. (Hrsg.), Cities after Socialism: Urban and Regional Change and Conflict in Post-Socialist Societies, S. 286-317. Oxford, Blackwell.

Tiosavlević R. (2011), Post-crisis development model of Serbia. In: SEER Journal for Labour and Social Affairs in Eastern Europe, 1, S. 7-15.

TODORIĆ J., RATKAJ I. (2011), Neighborhood perception as an indicator of gentrification in central zone of Belgrade. In: Journal of the Geographical Institute „Jovan Cvijić“, SASA, 61,3, S. 63-79

Tosić B. (2010), Introducing Belgrade. In: Krevs M., Djordjević D., Pichler-Milanović N. (Hrsg.), Challenges of spatial development of Ljubljana and Belgrade (= GeograFF, 8), S. 18-26. Ljubljana, Scientific Publishing House of the Faculty of Arts.

TSEnKova S. (2010), Informal settlements in post-communist cities: Diversity factors and patterns. In: Urbani izziv, 21, 2, S. 73-83.

UN-Habitat United Nations Human Settlements Programme (Hrsg.) (2003), The Challenge of Slums. Global Report on Human Settlements 2003. Nairobi.

UN-Habitat United Nations Human Settlements Programme (Hrsg.) (2006), Four Strategic Themes for the Housing Policy in Serbia. Belgrade. - http://www.unhabitat.org.rs/pdfs/ nhc_eng.pdf (letztmals aufgerufen am 15.4.2011)

URBANISTIČKI ZAVOD BEOGRADA (UZB) (2002), Nacrt generalnog urbanističkog plana Beograda 2021 [Entwurf eines städtebaulichen Masterplans von Belgrad 2021]. Beograd.

Vojković G., Devedžıć M. (2010), Demographic characteristics and processes in Belgrade. In: Krevs M., Duordjević D., Pichler-Milanović N. (Hrsg.), Challenges of spatial development of Ljubljana and Belgrade (= GeograFF, 8), S. 101-118. Ljubljana, Scientific Publishing House of the Faculty of Arts.

Vujosević M., Zeković S., Maričı́́ T. (2010), Post-socialist Transition and Spatial Development of Serbia. In: ManoJ J. (Hrsg.), Latest Trends on Urban Planning and Transportation. $3^{\text {rd }}$ WSEAS International Conference on Urban Planning and Transportation (UPT, 10), Corfu, S. 60-65. - http://www.wseas.us/books/2010/Corfu/UPT.pdf

Vujović S. (1986), O promenama u društvenoj i prostornoj strukturi Beograda [Über Veränderungen in der öffentlichen und räumlichen Struktur Belgrads]. In: Ideje, 16, 4, S. 113-134.

Vujović S. (1991), Stanovanje i društvene nejednakosti [Wohnen und öffentliche Ungleichheiten]. In: Popović U.M. (Hrsg.), Srbija krajem osamdesetih: sociološko istraživanje društvenih nejednakosti i neusklađenosti, S. 275-312. Beograd, Filozofksi fakultet - Institut za sociološka istraživanja.

Vujović S. (1995), Urbano i stambeno pitanje u svetlu svojinskih promena - skica za sociološku analizu [Die städtische und Wohnungsfrage im Lichte ihrer Veränderungen - Skizze zur soziologischen Analyse]. In: Luča, 12, 1-2, S. 272-278.

ZEGARAC Z. (1999), Illegal construction in Belgrade and the prospects for urban development planning. In: Cities, 16, 5, S. 365-370. 